\title{
Extensive Lymph Node Dissection Around the Left Laryngeal Nerve Achieved With Robot-assisted Thoracoscopic Esophagectomy
}

\author{
SATORU MOTOYAMA ${ }^{1,2,3^{*}}$, YUSUKE SATO ${ }^{1,2}$, AKIYUKI WAKITA ${ }^{1,2}$, YUTA KAWAKITA $^{1,2}$, \\ YUSHI NAGAKI ${ }^{1,2}$, KAZUHIRO IMAI $^{2}$ and YOSHIHIRO MINAMIYA ${ }^{2}$ \\ ${ }^{1}$ Esophageal Surgery, Akita University Hospital, Akita, Japan; \\ ${ }^{2}$ Thoracic Surgery, Akita University Graduate School of Medicine, Akita, Japan; \\ ${ }^{3}$ Comprehensive Cancer Control, Akita University Graduate School of Medicine, Akita, Japan
}

\begin{abstract}
Background/Aim: The potential advantages of robot-assisted thoracoscopic esophagectomy (RATE) have yet to be verified. This study focused on the degree of lymph node dissection around the left recurrent laryngeal nerve (RLN). Patients and Methods: A total of 21 consecutive patients who received RATE (RATE group) and 38 who received conventional thoracoscopic esophagectomy (TE) (TE group), both with extended lymphadenectomy, for thoracic squamous cell esophageal carcinoma, were enrolled in this study. We compared the numbers of lymph nodes dissected from the mediastinum, especially around the left RLN. Results: The median number of lymph nodes dissected from around the left $R L N$ was 6 in the RATE group and 4 in the TE group, and the ratio of the dissected lymph nodes around the left RLN to all lymph nodes was significantly higher in the RATE group ( $p=0.048$ ). Conclusion: Our findings indicate that a robotassisted surgical system enables for more extensive dissection of lymph nodes around the left RLN.
\end{abstract}

Thoracic squamous cell esophageal carcinoma (TESCC) is a highly aggressive malignancy with a poor prognosis that typically involves lymph node metastasis (1-3). For that reason, extensive lymph node dissection in the neck, mediastinum and abdomen, with or without perioperative chemo- or chemoradiotherapy, has been a mainstay of treatment for this type of cancer (1-4). Dissection of the lymph nodes in the mediastinum, especially around the left

Correspondence to: Satoru Motoyama, Esophageal Surgery, Akita University Hospital/Comprehensive Cancer Control, Akita University Graduate School of Medicine, 1-1-1 Hondo, Akita 0108543, Japan. Tel: +81 188846132, Fax: +81 188362615, e-mail: motoyama@doc.med.akita-u.ac.jp

Key Words: Esophagectomy, robot-assisted surgery, lymph node dissection. recurrent laryngeal nerve (RLN), is key to the curability of TESCC, but this requires a highly skillful technique to avoid left RLN palsy leading to postoperative laryngopharyngeal dysfunction or aspiration. In 2003, robot-assisted thoracoscopic esophagectomy (RATE) was developed (5). RATE provides the benefits of a 3D magnified view and articulated instruments, which enable precise dissection with 7 degrees of freedom of movement. Conventional thoracoscopic esophagectomy (TE) provides a significant advantage over open transthoracic surgery, provided the surgeon has the considerable experience necessary to perform this technique. RATE enables surgeons to easily and precisely maneuver without a prolonged training period.

The results from several cohort studies showed that RATE is feasible and safe and provides the benefits of a minimallyinvasive approach; however, its role and potential advantages have yet to be verified (6-8). Although a report suggests RATE reduces the incidence of vocal cord palsy and improves maneuverability when dissecting upper mediastinal lymph nodes, additional evidence is urgently needed (9). We, therefore, endeavored to add evidence to the data pool comparing conventional TE with RATE. In our first case series to compare conventional TE with RATE in the surgery for TSECC, we focused on the degree of lymph node dissection around the left RLN and assessed the advantage of RATE in the left lateral decubitus position.

\section{Patients and Methods}

Twenty-one consecutive patients who received RATE (RATE group) and 38 patients who received conventional TE (TE group), both with extended 2- or 3- field lymphadenectomy, for TESCC between Dec 2014 and Oct 2018 were enrolled. All patients were Eastern Cooperative Oncology Group performance status 0. To reduce heterogeneity in this cohort study, patients with synchronous double primary cancers, esophagogastric junctional cancer, or surgical reconstruction using colon or jejunum or pull-up through the subcutaneous route using a gastric tube were excluded. This study 
was approved by the Ethics Committee of Akita University Graduate School of Medicine (No. 1222).

RATE. All 21 patients provided informed consent to robotic surgery between Dec 2014 and Oct 2018. Ten patients who agreed to uninsured use of the da Vinci S or Si HD Surgical System underwent RATE between Dec 2014 and Mar 2018. Since Apr 2018, 11 patients have undergone esophagectomies using the da Vinci Si Surgical System in operations covered by insurance. All thoracic operations in the RATE group were performed by a consultant surgeon who had obtained the Japan Society for Endoscopic Surgery's skill qualification and the Japan Society for Esophageal Surgery's qualification. The patients were initially placed in the left lateral position under a combination of inhaled and intravenous anesthesia and a double-lumen endotracheal tube was used for single-lung ventilation (Figure 1). The right arm was raised 30 degrees cranially to expose the right axillar fossa, then tilted 15 degrees cranially and 15 degrees ventrally. Two assistant surgeons stood on the left side of the patient. The patient cart was docked 30 degrees clockwise relative to the craniocaudal axis of the patient (Figure 1). A 25-mm mini-thoracotomy was performed in the 4th intercostal space (ICS) on the anterior axillary line, carefully confirming the absence of pleural adhesion. A 12-mm trocar for the thoracoscope was inserted into the 6th ICS on the middle axillary line. Generally, another four trocars were inserted under thoracoscopic guidance: an 8-mm trocar in the 4th ICS on the posterior axillary line for the first arm of the robot, an 8-mm trocar in the 9th ICS on the posterior axillary line for the second arm, an 8 -mm trocar in the $2 \mathrm{nd}$ or $3 \mathrm{rd}$ ICS on the anterior axillary line for the third arm, and a $10.5-\mathrm{mm}$ trocar in the 8th ICS on the anterior axillary line for an assistant. The operator used Maryland bipolar forceps, a permanent cautery hook, monopolar curved scissors, Potts scissors, a small clip applier, a medium-large clip applier, and a large needle driver with the first arm; fenestrated bipolar forceps or Maryland bipolar forceps with the second arm; and Cadiere forceps with the third arm. We mainly used a forward-oblique viewing endoscope and often a forward-viewing endoscope.

Conventional TE. All 38 patients treated with conventional TE were operated on while in a left lateral position by five surgeons, including a surgeon consulting for insured medicine. As usual, a 25-mm mini-thoracotomy was performed in the 4th ICS on the anterior axillary line. Four $10.5-\mathrm{mm}$ trocars were inserted under thoracoscopic guidance: one in the 4th ICS on the posterior axillary line for the operator's left arm, one in the 5th ICS on the middle axillary line for the scope (for an assistant surgeon), one in the 7th ICS on the posterior axillary line for the operator's right arm, one in the 8th ICS on the anterior axillary line for an assistant.

Operative procedure. The thoracic part of the operation was nearly the same in the RATE and TE groups. The operation was begun by opening the mediastinal pleura on the dissected line. The arch of the azygos vein was divided and then ligated. The right bronchial artery was generally preserved after dissecting around it, though for technical reasons this artery was not preserved in some patients in the TE group. The lymph nodes around the right RLN could be dissected below the right subclavian artery. To dissect along the left RLN, the esophagus was pulled using tape to the vent-right lateral side to separate the esophagus from the trachea and main bronchus and to enlarge the limited space so as to increase the range of
Table I. Patient characteristics.

\begin{tabular}{|c|c|c|c|}
\hline & RATE (N=21) & $\mathrm{TE}(\mathrm{N}=38)$ & $p$-Value \\
\hline $\begin{array}{l}\text { Age, years, } \\
\text { median (range) }\end{array}$ & $63(44-76)$ & $66(49-75)$ & 0.151 \\
\hline \multicolumn{4}{|l|}{ Gender, n (\%) } \\
\hline Male & $19(90 \%)$ & $32(84 \%)$ & \multirow[t]{2}{*}{0.699} \\
\hline Female & $2(10 \%)$ & $6(16 \%)$ & \\
\hline \multicolumn{4}{|l|}{ Tumor location, $\mathrm{n}(\%)$} \\
\hline Upper & $6(29 \%)$ & $9(24 \%)$ & \multirow[t]{3}{*}{0.798} \\
\hline Middle & $7(33 \%)$ & $16(42 \%)$ & \\
\hline Lower & $8(38 \%)$ & $13(34 \%)$ & \\
\hline \multicolumn{4}{|l|}{$\mathrm{cT}, \mathrm{n}(\%)$} \\
\hline $\mathrm{T} 1 \mathrm{~b}$ & $5(24 \%)$ & $16(42 \%)$ & \multirow[t]{3}{*}{0.075} \\
\hline $\mathrm{T} 2$ & $5(24 \%)$ & $2(5 \%)$ & \\
\hline $\mathrm{T} 3$ & $11(52 \%)$ & $20(53 \%)$ & \\
\hline \multicolumn{4}{|l|}{$\mathrm{cN}, \mathrm{n}(\%)$} \\
\hline 0 & $8(38 \%)$ & $19(50 \%)$ & \multirow[t]{3}{*}{0.588} \\
\hline 1 & $10(48 \%)$ & $13(34 \%)$ & \\
\hline 2 & $3(14 \%)$ & $6(15 \%)$ & \\
\hline $\begin{array}{l}\text { Number of involved node, } \\
\text { median (range) }\end{array}$ & $1(0-4)$ & $0.5(0-3)$ & \multirow[t]{2}{*}{0.301} \\
\hline \multicolumn{3}{|l|}{ cStage, n (\%) } & \\
\hline IA & $4(19 \%)$ & $14(37 \%)$ & \multirow[t]{6}{*}{0.507} \\
\hline IB & $3(14 \%)$ & $2(5 \%)$ & \\
\hline IIA & $1(5 \%)$ & $3(8 \%)$ & \\
\hline IIB & $3(14 \%)$ & $2(5 \%)$ & \\
\hline IIIA & $7(33 \%)$ & $11(29 \%)$ & \\
\hline IIIB & $3(14 \%)$ & $6(16 \%)$ & \\
\hline \multicolumn{4}{|l|}{ Neoadjuvant therapy, n (\%) } \\
\hline Chemoradiotherapy & $12(57 \%)$ & $19(50 \%)$ & \multirow[t]{3}{*}{0.689} \\
\hline Chemotherapy & $0(0 \%)$ & $1(3 \%)$ & \\
\hline ER or None & $9(43 \%)$ & $18(47 \%)$ & \\
\hline
\end{tabular}

ER: Endoscopic resection.

movement. The thoracic duct was carefully preserved in T1-2 cancers, but was resected in $\mathrm{T} 3$ cancers. The pulmonary branches of the vagal nerves were preserved, and the intestinal branches were divided. For middle and lower thoracic esophageal cancers, the esophagus was divided at the level of the aortic arch by linear stapling; for upper thoracic esophageal cancers, it was divided in the supradiaphragmatic area. The lower posterior mediastinal lymph nodes were dissected from the pericardium, left pleura, descending aorta, and diaphragm.

Examination. Patients were observed for 2-48 months following surgery. We determined short-term surgical outcomes, including the number of lymph nodes dissected from the mediastinum, especially around the left RLN; the estimated blood loss during the entire surgery or during the thoracic part; and the incidence of postoperative complications, including vocal cord palsy.

Definitions. The clinical staging, including diagnosis of lymph node metastasis, was defined at a conference attended by radiologists, physicians and surgeons according to the International Union Against Cancer tumor-node-metastasis (TNM) Classification of Malignant Tumors (seventh edition) based on findings from contrast-enhanced computed tomography, esophagography, endoscopy, and systematic 


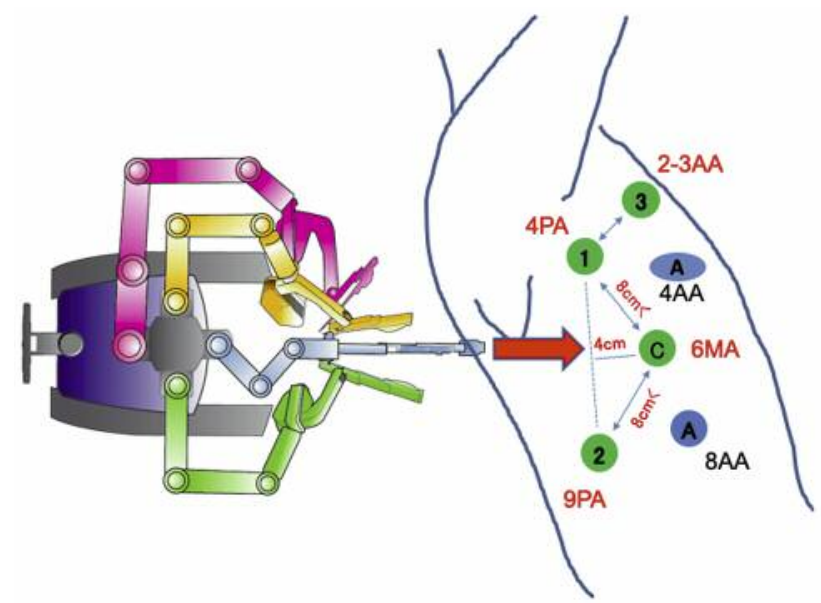

Figure 1. Port design for robot-assisted thoracoscopic esophagectomy in the left lateral decubitus position and a Dicking image. 1: 1st arm; 2: 2nd arm; 3: $3 \mathrm{rd}$ arm; A: assistant port; PA: posterior axillary line; MA: middle axillary line; AA: anterior axillary line.

$\left[{ }^{18} \mathrm{~F}\right]$ fluorodeoxyglucose-positron emission tomography/computed tomography (FDG-PET/CT) imaging. Regional nodes were considered positive for malignancy when they were round or ovoid shaped with short axes $\geq 10 \mathrm{~mm}$ in thin-sliced computed tomography. When ultrasonography imaging pattern in the neck lymph nodes showed welldefined boundary echo, weak or sonolucent internal echo or strong with notching internal echo, we considered there was metastasis in lymph node. The operating time for the thoracic phase of the surgery was defined as the time from the start of chest incision through closure of the trocar sites in the chest. Blood loss was estimated by weighing suctioned blood and gauze pieces with absorbed blood. Following the surgery, patients were admitted to the intensive care unit (ICU) without continuing mechanical ventilation. Surgical complications were evaluated using the Clavien-Dindo (CD) classification (10). Recurrent nerve palsy, with or without vocal cord movement disorder (None or $\leq \mathrm{CD} 1$ ), was observed by bronchoscopy during admission (nearly all patients were tested on post-operative day 2).

Statistical analysis. Continuous variables are presented as medians (minimum-maximum), and differences between the two groups were analyzed using the Mann-Whitney $U$-test. Categorized data were analyzed using the Pearson's Chi square test or Fisher's exact probability test. All statistical analyses were performed using JMP13 (SAS Institute Inc., Cary, NC, USA) and yielded two-sided $p$-values. Values of $p<0.05$ were considered statistically significant.

\section{Results}

The characteristics of the patients in the RATE and TE groups are summarized in Table I. There were no significant differences between the two groups with respect to age, sex, tumor location, tumor depth, lymph node or distant metastasis or clinical stage. The percentages of patients who received neoadjuvant therapy also did not differ. The operation times for the thoracic
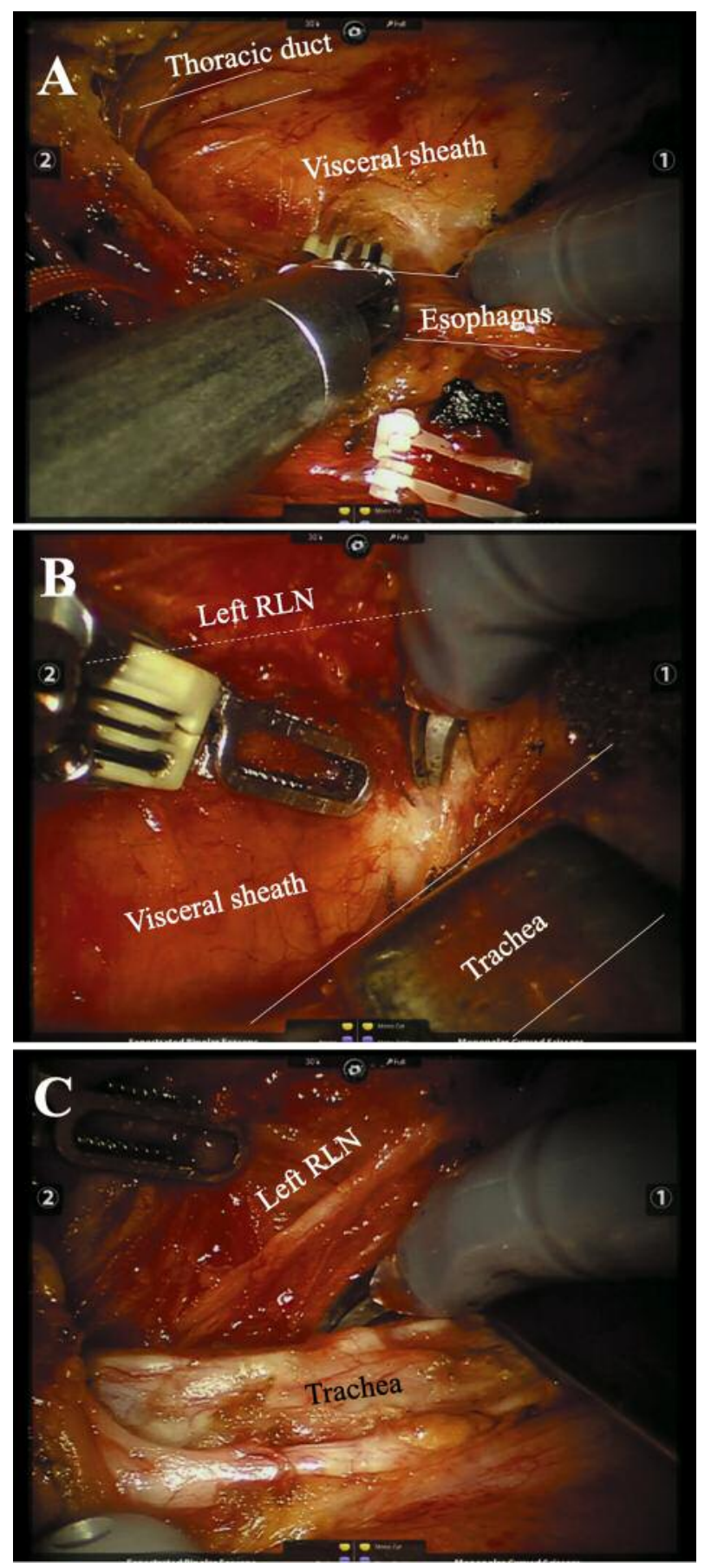

Figure 2. Operating views during robot-assisted thoracoscopic esophagectomy, with a special focus on its ability to offer adequate depth perception of the visceral sheath around the left recurrent laryngeal nerve during lymph node dissection.

part of the surgery were equal; however, blood loss during the thoracic surgery tended to be lower in the RATE group than the TE group, though the difference was not 
Table II. Surgical outcomes.

\begin{tabular}{|c|c|c|c|}
\hline & RATE $(\mathrm{N}=21)$ & $\mathrm{TE}(\mathrm{N}=38)$ & $p$-Value \\
\hline \multicolumn{4}{|l|}{ Area of lymph node dissection, $\mathrm{n}(\%)$} \\
\hline 2-field & $1(5 \%)$ & $2(5 \%)$ & \multirow{2}{*}{1.000} \\
\hline 3-field & $20(95 \%)$ & $36(95 \%)$ & \\
\hline \multicolumn{4}{|l|}{ Operation time (min), median (range) } \\
\hline All & $634(529-699)$ & $598.5(475-761)$ & 0.363 \\
\hline Intrathorax & $320(242-401)$ & $312.5(152-417)$ & 0.601 \\
\hline \multicolumn{4}{|l|}{ Blood-loss (ml), median (range) } \\
\hline All & $492(195-1591)$ & $385(177-3184)$ & 0.292 \\
\hline Intrathorax & $110(15-375)$ & $165(23-559)$ & 0.168 \\
\hline \multicolumn{4}{|c|}{ Number of dissected LN, median (range) } \\
\hline All & $52(36-104)$ & $59(35-97)$ & 0.222 \\
\hline Mediastinal & $23(11-41)$ & $20(7-68)$ & 0.371 \\
\hline Around the left laryngeal nerve & $6(0-15)$ & $4(0-12)$ & 0.212 \\
\hline \multicolumn{4}{|c|}{ Ratio of number of LN around the left laryngeal nerve, median (range) } \\
\hline Per all LN & $0.10(0.00-0.26)$ & $0.065(0.00-0.19)$ & $0.048 *$ \\
\hline Per mediastinal LN & $0.27(0.00-0.44)$ & $0.19(0.00-0.56)$ & 0.115 \\
\hline
\end{tabular}

LN: Lymph node; *statistically significant.

Table III. Surgical complications.

\begin{tabular}{|c|c|c|c|}
\hline & RATE (N=21) & $\mathrm{TE}(\mathrm{N}=38)$ & $p$-Value \\
\hline \multicolumn{4}{|c|}{ Left RLN palsy $(\mathrm{CD} \geq \mathrm{I}), \mathrm{n}(\%)$} \\
\hline Positive & $5(24 \%)$ & $18(47 \%)$ & \multirow[t]{2}{*}{0.098} \\
\hline Negative & $16(76 \%)$ & $20(53 \%)$ & \\
\hline \multicolumn{4}{|c|}{ Right RLN palsy $(\mathrm{CD} \geq \mathrm{I}), \mathrm{n}(\%)$} \\
\hline Positive & $2(10 \%)$ & $12(32 \%)$ & \multirow[t]{2}{*}{0.108} \\
\hline Negative & $19(90 \%)$ & $26(68 \%)$ & \\
\hline \multicolumn{4}{|c|}{ Pneumonia ( $\mathrm{CD} \geq \mathrm{IIIb}), \mathrm{n}(\%)$} \\
\hline Positive & $0(0 \%)$ & $0(0 \%)$ & \multirow[t]{2}{*}{1.000} \\
\hline Negative & $21(100 \%)$ & $38(100 \%)$ & \\
\hline \multicolumn{4}{|c|}{ Anastomotic leak $(C D \geq I), n(\%)$} \\
\hline Positive & $1(5 \%)$ & $3(8 \%)$ & \multirow[t]{2}{*}{1.000} \\
\hline Negative & $20(95 \%)$ & $35(92 \%)$ & \\
\hline \multicolumn{4}{|c|}{ Chyrothorax (CD $\geq \mathrm{IIIb}), \mathrm{n}(\%)$} \\
\hline Positive & $1(5 \%)$ & $1(3 \%)$ & \multirow[t]{2}{*}{1.000} \\
\hline Negative & $20(95 \%)$ & $37(97 \%)$ & \\
\hline
\end{tabular}

RLN: Recurrent laryngeal nerve; CD: Clavien-Dindo classification.

significant (Table II). The number of lymph nodes around the left RLN tended to be higher in the RATE group ( 6 in RATE and 4 in TE), but the difference was not significant (Table II). On the other hand, the ratio of the number of dissected lymph nodes around the left RLN to all dissected nodes was significantly higher in the RATE group than the TE group $(p=0.0482)$ (Table II). Left recurrent nerve palsy ( $\leq \mathrm{CD} 1)$ tended to be lower in the RATE group $(24 \%)$ than the TE group (47\%), but the difference was not significant (Table III). The incidences of other post-operative complications did not differ between the two groups.

\section{Discussion}

This study demonstrated that the ratio of the number of dissected lymph nodes around the left RLN to all dissected nodes was significantly higher in the RATE group than the conventional TE group, and there tended to be a lower incidence of left RLN palsy. Estimated blood loss during the thoracic surgery also tended to be lower in the RATE group, though the operation times during the thoracic part of the surgery did not differ between the two groups.

RATE has been covered by insurance in Japan since April 2018. To expand the use of robotic surgery in the future, two 
large problems that must be resolved are its extremely high cost and the lack of a clear advantage to using this system. van der Sluis et al. reported the first worldwide randomized controlled trial comparing RATE to transthoracic open esophagectomy in patients with esophageal cancer (11). They found that RATE was associated with a significantly lower overall incidence of surgery-related postoperative complications, less blood loss, and lower incidences of pulmonary and cardiac complications than open surgery. Postoperative pain during the first 14 postoperative days was lower after RATE than open surgery. Also, short-term quality of life after RATE was better, both at discharge and 6 weeks after discharge. On the other hand, there were no significant differences in oncologic outcome parameters.

The question then arises as to whether RATE is superior to conventional TE. Suda et al. reported that RATE significantly reduced the incidence of vocal cord palsy from $75 \%$ to $38 \%$ and hoarseness from $65 \%$ to $19 \%$ in their first case series comparing RATE to conventional TE (9). On the other hand, there were no significant differences in the numbers of dissected lymph nodes. Park et al. reported that the greatest benefit of RATE is the ability to perform radical dissection of lymph nodes around the left RLN without causing any stress, which requires delicate surgical manipulation within a limited space with a narrow range of movement (12). In a singlecenter, retrospective, propensity-matched study of 37 patients, Chao et al. recently found that compared to conventional TE, RATE enabled a higher lymph node yield along the left RLN without increasing morbidity (13). Moreover, Deng et al. reported that among 42 patients studied, RATE yielded significantly larger numbers of total dissected lymph nodes (21.9 vs. 17.8), right RLN lymph nodes (2.1 vs. 1.2), and abdominal lymph nodes (10.8 and 7.7) than conventional TE, with significantly less total blood loss than TE (97 and 161 $\mathrm{ml}$ ) (14). Postoperatively, there was no difference in the risk of major complications between the two groups.

In the present study, the surgical procedures were matched between the two groups, but the extent of lymph node dissection around the left RLN was higher in the RATE group, which showed a higher ratio of the number of dissected lymph nodes around the left RLN to all dissected nodes. Lymph node dissection around the left RLN is difficult, and dissection of nodes situated ventrally to the left RNL is a particularly challenging surgical procedure. With conventional TE, the limited flexibility of the instrument precludes tension-free dissection. RLN injury can be caused by contusion, excessive stretching, and thermal burn during dissection around the nerve. To reduce these injuries, we tried to suspend the esophagus using tape with the goal of expanding the space between the esophagus and trachea. However, this maneuver may itself excessively stretch the RLN. Unfortunately, therefore, left RNL palsy can still occur.
The advantages of robot-assisted surgery using the da Vinci are 1) depth perception facilitated by a 3D image, 2) maneuverability of the forceps with highly flexible joints, and 3) a stable surgical field for the surgeon and camera mediated by anti-shake functionality. More specifically, for lymph node dissection around the left RLN, the following advantages were noted. The 3D self-controlled magnified view enabled better visualization of the area, thanks to its ability to offer adequate depth perception of the visceral sheath and vascular sheath around the left RLN (Figure 2) (15). In addition, use of a self-controlled third arm and a tremor filtering function enabled us to achieve fine tension and countertraction during dissection in this area.

This study has several limitations: the number of enrolled patients was small, as this was our first case series; it was a non-randomized comparative analysis; only short-term results were determined. Because RATE is still at a development stage, surgeons have to learn through the early phase of studies.

In summary, our findings indicate that a robot-assisted surgical system enables for a more extensive dissection of lymph nodes around the left RLN without increasing the incidence of left RLN palsy. RATE has the potential to contribute to more precise surgery for thoracic esophageal cancer in the future.

\section{Conflicts of Interest}

None of the Authors have any conflicts of interest to declare regarding this study.

\section{Authors' Contributions}

Guarantors of integrity of entire study and Study concepts: Motoyama S and Minamiya Y; Study design or data acquisition or data analysis: Motoyama S, Sato Y, Wakita A, Kawakita Y, Nagaki Y; Statistical analysis, and manuscript editing, Motoyama S, Imai K.

\section{References}

1 Tachimori Y, Ozawa S, Numasaki H, Ishihara R, Matsubara H, Muro K, Oyama T, Toh Y, Udagawa H, Uno T and Registration Committee for Esophageal Cancer of the Japan Esophageal Society: Comprehensive registry of esophageal cancer in Japan, 2010. Esophagus 14(3): 189-214, 2017. PMID: 28725168, DOI: 10.1007/s 10388-017-0578-4

2 Tachimori Y, Ozawa S, Numasaki H, Matsubara H, Shinoda M, Toh Y, Udagawa H, Fujishiro M, Oyama $T$, Uno $T$ and Registration Committee for Esophageal Cancer of the Japan Esophageal Society: Efficacy of lymph node dissection by node zones according to tumor location for esophageal squamous cell carcinoma. Esophagus 13(1): 1-17, 2016. PMID: 26752982, DOI: $10.1007 / \mathrm{s} 10388-015-0515-3$

3 Chen MF, Yang YH, Lai CH, Chen PC and Chen WC: Outcome of patients with esophageal cancer: a nationwide analysis. Ann Surg Oncol 20(9): 3023-3030, 2013. PMID: 23525703, DOI: 10.1245/s 10434-013-2935-4 
4 Wong IYH and Law S: Surgery in the era of neoadjuvant therapy for cancer of the esophagus. Esophagus 13(2): 105-109, 2016. DOI: $10.1007 / \mathrm{s} 10388-016-0523-y$

5 van Hillegersberg R, Boone J, Draaisma WA, Broeders IA, Giezeman MJ and Borel Rinkes IH: First experience with robotassisted thoracoscopic esophagolymphadenectomy for esophageal cancer. Surg Endosc 20(9): 1435-1439, 2006. PMID: 16703427, DOI: 10.1007/s00464-005-0674-8

6 Boone J, Schipper ME, Moojen WA, Borel Rinkes IH, Cromheecke GJ and van Hillegersberg R: Robot-assisted thoracoscopic oesophagectomy for cancer. Br J Surg 96(8): 878886, 2009. PMID: 19591168, DOI: 10.1002/bjs.6647

7 Kim DJ, Hyung WJ, Lee CY, Lee JG, Haam SJ, Park IK and Chung KY: Thoracoscopic 20 esophagectomy for esophageal cancer: feasibility and safety of robotic assistance in the prone position. J Thorac Cardiovas Surg 139(1): 53-59, 2010. PMID: 19660280, DOI: 10.1016/j.jtcvs.2009.05.030

8 Puntambekar SP, Rayate N, Joshi S and Agarwal G: Robotic transthoracic esophagectomy in the prone position: experience with 32 patients with esophageal cancer. J Thorac Cardiovac Surg 142(5): 1283-1284, 2011. PMID: 21530982, DOI: 10.1016/ j.jtcvs.2011.03.028

9 Suda K, Ishii Y, Kawamura $\mathrm{Y}$ and Inaba K, Kanaya S, Teramukai S, Satoh S and Uyama I: Robot-assisted thoracoscopic lympha-denectomy along the left recurrent laryngeal nerve for esophageal squamous cell carcinoma in the prone 5 position: technical report and short-term outcomes. World J Surg 36(7): 1608-1616, 2012. PMID: 22392356, DOI: $10.1007 / \mathrm{s} 00268-012-1538-8$

10 Clavien PA, Barkun J, de Oliveira ML, Vauthey JN, Dindo D, Schulick RD, de Santibañes E, Pekolj J, Slankamenac K, Bassi C, Graf R, Vonlanthen R, Padbury R, Cameron JL and Makuuchi M: The Clavien-Dindo classification of surgical complications: fiveyear experience. Ann Surg 250(2): 187-196, 2009. PMID: 19638912, DOI: 10.1097/SLA.0b013e3181b13ca2
11 van der Sluis PC, van der Horst S, May AM and Schippers C, Brosens LAA, Joore HCA, Kroese CC, Haj Mohammad N, Mook S, Vleggaar FP, Borel Rinkes IHM, Ruurda JP and van Hillegersberg R: Robot-assisted minimally invasive thoracolaparoscopic esophagectomy versus open transthoracic esophagectomy for resectable esophageal cancer: A randomized controlled trial. Ann Surg, 2018. PMID: 30308612, DOI:10.1097/SLA.00000 00000003031

12 Park S, Hwang Y, Lee H J and Park IK, Kim YT and Kang CH: Comparison of robot-assisted esophagectomy and thoracoscopic esophagectomy in esophageal squamous cell carcinoma. J Thorac Dis 8(10): 2853-2861, 2016. PMID: 27867561, DOI: $10.21037 / \mathrm{jtd} .2016 .10 .39$

13 Chao YK, Hsieh M, Liu YH and Liu HP: Lymph node evaluation in robot-assisted versus video-assisted thoracoscopic esophagectomy for esophageal squamous cell carcinoma: A propensity-matched analysis. World J Surg 42(2): 590-598, 2018. PMID: 28801820, DOI: 10.1007/s00268-017-4179-0

14 Deng HY, Huang WX, Li G and Li SX, Luo J, Alai G, Wang Y, Liu LX and Lin YD: Comparison of short-term outcomes between robot-assisted minimally invasive esophagectomy and video-assisted minimally invasive esophagectomy in treating middle thoracic esophageal cancer. Dis Esophagus 31(8): 1-7, 2018. PMID: 29538633, DOI: 10.1093/dote/doy012

15 Tokairin Y, Nakajima Y, Kawada Kand Hoshino A, Okada T, Ryotokuji T, Okuda M, Kume Y, Kawamura Y, Yamaguchi K, Nagai K, Akita K and Kinugasa Y: Histological study of the thin membranous structure made of dense connective tissue around the esophagus in the upper mediastinum. Esophagus 15(4): 272280, 2018. PMID: 29948479, DOI: 10.1007/s10388-018-0625-9

Received February 3, 2019

Revised February 14, 2019

Accepted February 18, 2019 